\title{
Group voice enhancement for professors
}

\author{
Natalie Schaeffer* \\ Brooklyn College, Speech and Hearing Clinic, Brooklyn, NY 11210, USA
}

\begin{abstract}
A number of professors at the college complained, that although they do not have voice difficulty, they have problems maintaining an appropriate voice when speaking for four hours or more (at a time) during their lectures. The professors reported that their voices become strained, and they cannot maintain appropriate intensity, making it difficult for the students to hear the content of their lecture. Thus, the Voice Enhancement Group was formed for the professors to practice using their voices productively and efficiently. The professors learned appropriate vocal techniques, practicing them in structured tasks leading to spontaneous speech.
\end{abstract}

\section{General instructions to maintain a healthy voice Voice enhancement therapy}

1. Keep your body aligned to allow an easy and efficient flow of breath for voice production.

2. To coordinate respiration and phonation, take in a regular breath (not a deep breath) through your mouth and produce a sentence while simultaneously pulling in your abdominal muscles (for support) upon the production of the sentence/sentences (training is needed).

3. Pause completely between sentences to allow the next breath to be inhaled (automatically or purposely) to have breath for the next sentence. Do not raise your shoulders when inhaling.

4. Speak/articulate at a normal pace, not rapidly, so that the vocal effort lands on the articulators, not on your vocal folds. For example, if you articulate the word "make" the production of $/ \mathbf{m} /$ should land on your lips even though the vocal folds are creating the sound. Good articulation should take the effort off the larynx.

5. Vary your pitch for meaning and to allow the vocal folds to elongate and shorten intermittently, so that they do not continuously remain in the same configuration.

6. Do not shout to increase vocal intensity. Pausing appropriately and pulling in your abdominal muscles smoothly when speaking allows for a stronger voice and vocal support, reducing strain while appropriately increasing vocal intensity.

7. Use an easy onset of initial vowels-no glottal attacks (forceful closure of the vocal folds).

8. Drink at least 4-6 glasses of water a day to hydrate your body and phonatory system.

\section{Specific outline of voice therapy for the professors}

The professors sat on one side of a long table, and the students sat facing them on the other side, while Dr. Schaeffer sat at the head of the table guiding the steps of therapy as follows:

1. The professors were initially told to stand up, sip in a small breath, and produce the following vowels (one by one /ah, ee, oo/) while simultaneously pulling in their abdominal muscles to remove any strain from the larynx/vocal folds.

2. Once the professors correctly used their breath and abdominal muscles to produce vowels and then one-syllable words (initial voiced consonants enhanced voices which were breathy (e.g., make, more, neck, look, get, bake, good, jump), and initial voiceless consonants (e.g., take, cook, put, ) made it easier to improve tight voices. Eventually, both initial voiced and voiceless consonants were used, as appropriate.

3. These words were then practiced in two- and three-word phrases (e.g., initial voiced consonants--get more, look good; initial voiceless consonants--take care, pick two).

4. The phrases are then put together with pausing between them (e.g., Get more/good milk).

5. Three-word phrases with appropriate consonants and pausing between the phrases were next.

6. When the client is ready, mix voiced and voiceless consonants (e.g., more cake, good cake), and three word phrases with the appropriate sounds and pausing between the phrases.

7. Once the client's voice improves on these phrases, co-articulation is taught. Co-articulation consists of connecting the final consonant with a following initial vowel so the vocal folds do not bang together on the initial vowel, for example (come on), (pick up).

8. These phrases can be put into a sentence eventually (e.g., Please come on).

9. Initial vowels must be addressed to prevent glottal attacks. Slightly elongate the initial vowel (iiiin) and then shorten the vowel, maintaining the smooth flow of the vowel in the word.

${ }^{\star}$ Correspondence to: Natalie Schaeffer, D.A. CCC-SLP, Professor, Brooklyn College, Speech and Hearing Clinic - Boylan 4400, 2900 Bedford Ave., Brooklyn, NY 11210, USA, Tel: 718.951.5282; E-mail: natalies@brooklyn.cuny.edu

Received: September 17, 2020; Accepted: October 12, 2020; Published: October 16,2020 
10.Practice rhythmic verse (e.g., reciting short songs, not singing) making it easy to incorporate all the techniques above, pausing at the periods and commas.

11. Vary the pitch for meaning so that the vocal fold do not remain in the same pitch.

Sing, sing a song

Sing out loud.

Sing out strong.

Sing of good things

Not bad, Sing of happy, not sad.

This kind of content (i.e., the poem above) allows the client to practice all the techniques in a structured task: pausing appropriately between phrases, using the abdominal muscles to support voice, easy onset of vowels (no glottal attacks). Once the client has practiced these techniques in structures tasks, as noted above, the content can be extended in longer sentences, and in role playing in spontaneous speech, always pausing appropriately to replenish breath supply. For example, you can introduce short spontaneous tasks at first such as the following: Ask "What would happen if you left your keys in the car?" The client could answer "If I left my keys in the car, someone could steal the car." For this answer, easy onset of vowels, co-articulation, pausing at commas and periods, and appropriate pulling in the abdominal muscles for breath support can easily be practiced. Role playing with the professors was incorporated. For example, the topic of "going on a trip," each professor gave a short suggestion (using all the techniques) learned.

Once the client can naturally use the techniques, these techniques are not hard to incorporate into structured spontaneous speech, and the voice will not be abused. The professors can use the techniques in longer content and when teaching a class. During the therapy sessions, a tape recorder was used to have the professors analyze their own responses in terms of their voices. Describing sequencing pictures is also a good activity to practice pausing appropriately (i.e., at the end of a sentence and then in spontaneous speech).

One has to build up to longer content by using techniques in structured tasks and then towards spontaneous speech until supported speech becomes automatic. The professors did very well, and the students became proficient in providing therapy tasks and feedback.

Copyright: (C2020 Schaeffer N. This is an open-access article distributed under the terms of the Creative Commons Attribution License, which permits unrestricted use, distribution, and reproduction in any medium, provided the original author and source are credited. 